This item was submitted to Loughborough's Research Repository by the author.

Items in Figshare are protected by copyright, with all rights reserved, unless otherwise indicated.

\title{
Acoustic propulsion of a small, bottom-heavy sphere
}

PLEASE CITE THE PUBLISHED VERSION

https://doi.org/10.1017/jfm.2020.401

PUBLISHER

Cambridge University Press (CUP)

\section{VERSION}

AM (Accepted Manuscript)

\section{PUBLISHER STATEMENT}

This article has been published in a revised form in Journal of Fluid Mechanics https://doi.org/10.1017/jfm.2020.401. This version is published under a Creative Commons CC-BY-NC-ND. No commercial re-distribution or re-use allowed. Derivative works cannot be distributed. () The Authors.

\section{LICENCE}

CC BY-NC-ND 4.0

\section{REPOSITORY RECORD}

Nadal, Francois, and Sebastien Michelin. 2020. "Acoustic Propulsion of a Small, Bottom-heavy Sphere". Loughborough University. https://hdl.handle.net/2134/12290291.v1. 


\title{
Acoustic propulsion of a small bottom-heavy sphere
}

\author{
François $\mathrm{Nadal}^{1} \dagger$ and Sébastien Michelin ${ }^{2}$ \\ ${ }^{1}$ Wolfson School of Mechanical, Electrical and Manufacturing Engineering, \\ Loughborough University, LE11 3TU, Loughborough, United Kingdom \\ ${ }^{2}$ LadHyX - Département de Mécanique, CNRS - École Polytechnique, \\ Institut Polytechnique de Paris, 91128 Palaiseau, France
}

(Received xx; revised $\mathrm{xx}$; accepted $\mathrm{xx}$ )

We present here a comprehensive derivation for the speed of a small bottom-heavy sphere forced by a transverse acoustic field and thereby establish how density inhomogeneities may play a critical role in acoustic propulsion. The sphere is trapped at the pressure node of a standing wave whose wavelength is much larger than the sphere diameter. Due to its inhomogeneous density, the sphere oscillates in translation and rotation relative to the surrounding fluid. The perturbative flows induced by the sphere's rotation and translation are shown to generate a rectified inertial flow responsible for a net mean force on the sphere that is able to propel the particle within the zero-pressure plane. To avoid an explicit derivation of the streaming flow, the propulsion speed is computed exactly using a suitable version of the Lorentz reciprocal theorem. The propulsion speed is shown to scale as the inverse of the viscosity, the cube of the amplitude of the acoustic field and is a non trivial function of the acoustic frequency. Interestingly, for some combinations of the constitutive parameters (fluid to solid density ratio, moment of inertia and centroid to center of mass distance), the direction of propulsion is reversed as soon as the frequency of the forcing acoustic field becomes larger than a certain threshold. The results produced by the model are compatible with both the observed phenomenology and the orders of magnitude of the measured velocities.

\section{Introduction}

Controlled propulsion of microscopic objects in viscous flows has recently attracted much attention for its potential biomedical applications such as drug transport and delivery (Nelson et al. 2010; Sundararajan et al. 2008; Burdick et al. 2008) or analytical sensing in biological media (Campuzano et al. 2011; Wu et al. 2010). Self-propulsion in viscous flows requires temporal and spatial symmetry-breaking (Purcell 1977; Lauga \& Powers 2009). Based on that principle, many different mechanisms have been proposed to achieve propulsion of small rigid objects (see the reviews of Ebbens \& Howse 2010; Wang et al. 2013) and they generally belong to either of the two following categories.

The first and most classical group exploits an externally-applied directional field, that effectively breaks the symmetry of the system at a scale much larger than the particle size, and drives the object in a specific direction. Electrophoresis (Smoluchowsky 1921) and diffusiophoresis (Anderson 1989) both fall in this first group, and result from the application of macroscopic electric or chemical gradients. The alternative approach relies on the local interaction of the particle with its close environment. Taking advantage of

$\dagger$ Email address for correspondence: F.R.Nadal@lboro.ac.uk 
its own asymmetry, the particle converts locally the energy provided by a non-directional forcing field to break symmetry and self-propel.

For instance, catalytic bimetallic micro-rods can propel themselves (self-electrophoresis) at high velocities (up to $10 \mu \mathrm{m} \mathrm{s}^{-1}$ ) by oxidizing hydrogen peroxide and exploiting the resulting self-generated local electric fields (see e.g. Paxton et al. 2004; Ibele et al. 2007; Ebbens \& Howse 2011). For non-ionic solutes, the concentration gradient can also trigger a net motion of the particle through self-diffusiophoresis (Pavlick et al. 2011, 2013; Golestanian et al. 2007; Cordova-Figueroa \& Brady 2008). Similarly, autonomous propulsion can be achieved by taking advantage of self-thermophoresis effects (Jiang et al. 2010; Baraban et al. 2012; Qian et al. 2013). Unfortunately, electrochemicallyand thermally-based methods are not bio-compatible as a result of the inherent toxicity of the involved fuels (hydrogen peroxide, hydrazine) or of the required temperature differences.

Alternatively, acoustic fields may be used to achieve autonomous motion in biofluids, which explains the increasing interest of the scientific community in this type of propulsion method. Wang et al. (2012) demonstrated experimentally that bi-metallic rods with asymmetric shape or composition were able to self-propel with velocities up to 200 $\mu \mathrm{ms}^{-1}$ when trapped in the nodal plane of an acoustic resonator. This pioneering work was soon extended to various configurations and geometries, and self-acoustophoresis of magnetic clusters or asymmetric particles was thus reported (Sabrina et al. 2018; Ahmed et al. 2014, 2016). Kaynak et al. (2017) showed that bio-inspired acoustic micro-swimmers with dedicated shapes were even able to reach velocities up to $1200 \mu \mathrm{m} \mathrm{s}^{-1}$. Although the prescribed acoustic field from which self-propulsion originates is directional, selfpropulsion is achieved in a plane orthogonal to the excitation and its direction is not set by the external driving in constrast, for instance, with classical electrophoretic migrations of particles along the imposed forcing.

Since the seminal work of Wang et al. (2012), acoustic propulsion has been repeatedly ascribed to the streaming flows self-generated by the particle's periodic motion with respect to its fluid environment of small yet finite inertia (Riley 1966; Nadal \& Lauga 2014; Collis et al. 2017; Kaynak et al. 2017). To analyze the potential role of a particle's asymmetric shape on its ability to self-propel, Nadal \& Lauga (2014) first derived an integral form of the steady axial velocity of an acoustically-forced near-sphere, exploiting the absence of rotation of the particle at leading order in the particle's asymmetry as suggested by Zhang \& Stone (1998). Lippera et al. (2019) recently showed however that this configuration did not actually yield any propulsion at leading order and that higherorder corrections in the particle's asymmetry were necessary to obtain a rectified effect. Collis et al. (2017) considered the opposite case of an asymmetric (in density or shape) dumbbell of large aspect ratio, and showed that the propelling streaming flow actually arose from the inertial coupling between the viscous flows respectively generated by the particle's translation and rotation, suggesting that acoustically-generated rotation of the particle was just as essential as its periodic translation in order to obtain acoustic propulsion.

Inspired by this observation, we analyse here how acoustic self-propulsion of a geometrically-symmetric particle (i.e. a sphere) may be achieved when its non-uniform density results in a combined translation and rotation under the effect of the acoustic forcing. We thus present the full analytical derivation of the leading order propulsion velocity of a non homogeneous sphere trapped at the nodal plane of a resonator. The center of mass and centroid of the sphere do not coincide anymore, and as a result an inertial torque is imposed on the acoustically-forced sphere driving a combination of translational and rotational motions. We demonstrate that spherical particles may thus 


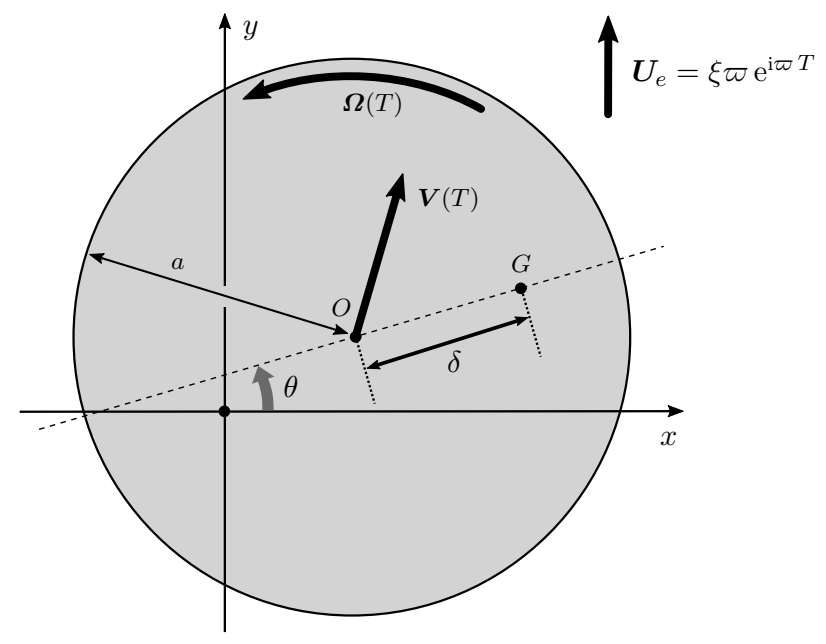

FiguRE 1. Oscillations of a bottom-heavy sphere forced by a uniform external oscillating flow. $\xi$ and $\varpi$ are the displacement amplitude and frequency of the forcing acoustic field. The radius of the sphere is denoted by $a$, and $\delta$ refers to the centroid-to-center of mass distance $O G$.

self-propel thanks to a symmetry-breaking in the hydrodynamic stress resulting from the inertial coupling of the viscous flows associated to the particle's combined translation and rotation, as suggested by Collis et al. (2017).

The paper is organized as follows. Section 2 is devoted to the derivation of the linear translational and rotational viscous responses of a non-homogeneous sphere to the transverse acoustic forcing. The leading order inertial propulsion velocity of such a sphere is obtained in section 3 by means of a suitable version of the Lorentz reciprocal theorem. The physical relevance of this model to experimental observations is then discussed in $\S 4$. Finally, our main findings are summarised in $\S 5$.

\section{Acoustically forced dynamics of a sphere in a viscous fluid}

\subsection{Configuration and main assumptions}

We consider here the dynamics of a solid sphere of radius $a$ and mean density $\rho_{s}$ forced by a viscous periodic flow of density $\rho$ and kinematic viscosity $\nu$. The density distribution of the solid sphere is not homogeneous, so that the center of mass $G$ departs from its centroid $O$ (Figure 1). The mass and volume of the sphere are respectively $m_{s}=\rho_{s} \mathcal{V}_{s}$ and $\mathcal{V}_{s}=(4 / 3) \pi a^{3}$.

The forcing (acoustic) flow $\boldsymbol{U}_{e}$ is uniform, harmonic of frequency $\varpi$ and directed along the $y$-direction. This configuration corresponds to a sphere of size $a$ trapped at the pressure node of a standing acoustic wave of wave vector $\boldsymbol{k}=k \mathbf{e}_{y}$ in the limit $k a \ll 1$ (with $\left(\mathbf{e}_{x}, \mathbf{e}_{y}, \mathbf{e}_{z}\right)$ are the Cartesian unit vectors). In such a case, the incident flow can be considered as locally incompressible and to depend only on $y$. We consider in the following that the external forcing flow is uniform and takes the simple harmonic form

$$
\boldsymbol{U}_{e}=\hat{\boldsymbol{U}}_{e} \mathrm{e}^{\mathrm{i} \varpi T}=\xi \varpi \mathrm{e}^{\mathrm{i} \varpi T} \mathbf{e}_{y},
$$

where $\xi$ is the amplitude of the fluid particles' displacement in the $y$-direction and is assumed to be much smaller than the particle's size $a$, so that $\varepsilon=\xi / a \ll 1$.

The offset of the sphere's centroid and center of mass is characterized by $\boldsymbol{\delta}=\mathbf{O G}=\delta \boldsymbol{d}$, where $\boldsymbol{d}=\cos \theta \mathbf{e}_{x}+\sin \theta \mathbf{e}_{y}$ (Figure 1). The velocity of $O$ and $G$ in the laboratory 
reference frame are denoted by $\boldsymbol{V}_{O}$ and $\boldsymbol{V}_{G}$ and the angular velocity $\boldsymbol{\Omega}$ of the sphere is aligned with $z$-direction: $\boldsymbol{\Omega}=\dot{\theta} \mathbf{e}_{z}$ (i.e. we assume that the sphere's density distribution is symmetric with respect to the $(O x y)$-plane). In the following, we make the additional assumption that the velocity of the particle is periodic for the zero-mean forcing flow $\boldsymbol{U}_{e}(t)$ considered (note however, that its mean value is not necessarily zero so as to allow for self-propulsion regimes).

The objective of the present section is to derive the response of the sphere to the external flow in an unsteady Stokesian framework, where inertia of the fluid is negligible, but that of the solid particle is not.

\subsection{Momenta conservation}

The conservation of momentum in the (Galilean) frame of reference can be written

$$
m_{s} \dot{\boldsymbol{V}}_{G}=\boldsymbol{F}+\boldsymbol{F}_{p},
$$

where the total force experienced by the sphere is the sum of the hydrodynamic force $\boldsymbol{F}$ due to the relative velocity between the sphere and the surrounding fluid, and of the pressure force $\boldsymbol{F}_{p}=\rho \mathcal{V}_{s} \dot{\boldsymbol{U}}_{e}$ arising from the external pressure gradient that sets the fluid in motion. Such a distinction is justified by the form of the viscous drag experienced by a solid sphere oscillating in an uniformly oscillating flow presented by Kim \& Karrila (2005). Note that assuming that the velocity of the particle is periodic in time immediately implies that the time-average of $\boldsymbol{F}(t)$ is zero.

Using $\boldsymbol{V}_{G}=\boldsymbol{V}_{O}-\boldsymbol{\delta} \times \boldsymbol{\Omega}$, and noting $\boldsymbol{V}=\boldsymbol{V}_{O}-\boldsymbol{U}_{e}$ the velocity of the sphere relative to the oscillating fluid, the previous equation can be rewritten

$$
\rho_{s} \mathcal{V}_{s} \dot{\boldsymbol{V}}=m_{s}[(\boldsymbol{\Omega} \times \boldsymbol{\delta}) \times \boldsymbol{\Omega}+\boldsymbol{\delta} \times \dot{\boldsymbol{\Omega}}]+\boldsymbol{F}+\left(\rho-\rho_{s}\right) \mathcal{V}_{s} \dot{\boldsymbol{U}}_{e}
$$

where the last term is the effective buoyancy force.

Similarly, the conservation of angular momentum can be written about the center of mass $G$,

$$
I_{G} \dot{\Omega}=\boldsymbol{L}_{G},
$$

where $I_{G}$ is the moment of inertia of the sphere about the $(G, z)$-axis and $\boldsymbol{L}_{G}$ is the total torque experienced by the sphere at its center of mass.

The sphere is rigid, thus $\boldsymbol{L}_{G}=\boldsymbol{L}+\boldsymbol{G} \boldsymbol{O} \times\left(\boldsymbol{F}+\boldsymbol{F}_{p}\right)$, with $\boldsymbol{L}$ is the hydrodynamic torque about the geometric center $O$, and Eq. (2.4) finally becomes

$$
I_{G} \dot{\boldsymbol{\Omega}}=\boldsymbol{L}-\boldsymbol{\delta} \times\left(\boldsymbol{F}+\boldsymbol{F}_{p}\right) .
$$

\subsection{Dimensionless forms of the conservation laws}

In the following, using $U_{e}=\xi \varpi$ and $\varpi^{-1}$ as reference velocity and time scales, respectively, yields the non-dimensional form of Eqs. (2.3) and (2.5) (using lower-case letters for dimensionless variables)

$$
\begin{gathered}
\dot{\boldsymbol{v}}=\varepsilon^{-1} \alpha[(\boldsymbol{\omega} \times \boldsymbol{d}) \times \boldsymbol{\omega}+\boldsymbol{d} \times \dot{\boldsymbol{\omega}}]+\left(\frac{3}{4 \pi} \frac{\beta}{\lambda^{2}}\right) \boldsymbol{f}+(\beta-1) \boldsymbol{f}_{e}, \\
\dot{\boldsymbol{\omega}}=\left(\frac{15}{8 \pi} \frac{\beta}{\lambda^{2} I}\right) \boldsymbol{l}-\varepsilon\left[\left(\frac{15}{8 \pi} \frac{\alpha \beta}{\lambda^{2} I}\right)(\boldsymbol{d} \times \boldsymbol{f})+\left(\frac{5}{2} \frac{\alpha \beta}{I}\right)\left(\boldsymbol{d} \times \boldsymbol{f}_{e}\right)\right],
\end{gathered}
$$

with $\boldsymbol{f}_{e}=\mathrm{i} \mathrm{e}^{\mathrm{i} t} \mathbf{e}_{y}$ the fluctuating forcing.

In the above equations, the dimensionless moment of inertia $I=I_{G} / I_{0}$ is the ratio 
between the actual moment of inertia $I_{G}$ and $I_{0}=(2 / 5) m_{s} a^{2}$, the moment of inertia of a homogeneous sphere with the same mean density with respect to its centre. Further, $\alpha=\delta / a$ is the relative geometric offset of the particle's center of mass and thus characterizes its non-homogeneity, $\beta=\rho / \rho_{s}$ is the fluid-to-solid average density ratio and $\lambda=\left(a^{2} \varpi / \nu\right)^{1 / 2}$ is the ratio between the radius of the sphere and the viscous penetration length (i.e. $\lambda^{2}$ is the reduced frequency of actuation). It should be noted that the $\varepsilon^{-1}$ factors are associated with the choice of characteristic velocity scale.

\subsection{Harmonic response in the unsteady Stokes limit}

The non-dimensional forcing field $\boldsymbol{f}_{e}=f_{e} \mathbf{e}_{y}$ with $f_{e}=\mathrm{ie} \mathrm{i}^{\mathrm{i} t}$ is $O\left(\varepsilon^{0}\right)$ and harmonic; as a result, for $\varepsilon \ll 1$, the leading order dynamics is obtained by noting that $v_{y}=O(1)$ while $v_{x}$ and $\theta$ are $O(\varepsilon)$ :

$$
\begin{gathered}
\dot{v}_{x}=\alpha \varepsilon^{-1}\left(\dot{\theta}^{2}+\ddot{\theta} \theta\right)+\left(\frac{3}{4 \pi} \frac{\beta}{\lambda^{2}}\right) f_{x}, \\
\dot{v}_{y}=-\alpha \varepsilon^{-1} \ddot{\theta}+\left(\frac{3}{4 \pi} \frac{\beta}{\lambda^{2}}\right) f_{y}+(\beta-1) f_{e}, \\
\ddot{\theta}=\left(\frac{15}{8 \pi} \frac{\beta}{\lambda^{2} I}\right) l-\varepsilon\left[\left(\frac{15}{8 \pi} \frac{\alpha \beta}{\lambda^{2} I}\right) f_{y}+\left(\frac{5}{2} \frac{\alpha \beta}{I}\right) f_{e}\right] .
\end{gathered}
$$

In the unsteady Stokes limit, the total viscous force and torque on the sphere are obtained by superimposing that induced by the sphere's translation and rotation independently. By symmetry, the force induced by the sphere's rotation and the torque (about $O$ ) induced by the sphere's translation are both identically zero. Therefore, considering the form of the system (2.8)-(2.10) and according to Kim \& Karrila (2005), one can write

$$
v_{x}=\varepsilon \hat{v}_{0, x} \mathrm{e}^{2 \mathrm{i} t}, \quad v_{y}=\hat{v}_{0, y} \mathrm{e}^{\mathrm{i} t}, \quad \theta=\varepsilon \hat{\theta}_{0} \mathrm{e}^{\mathrm{i} t}
$$

and

$$
f_{x}=-\Delta_{2} v_{x}, \quad f_{y}=-\Delta_{1} v_{y}, \quad l=-\Lambda_{1} \dot{\theta},
$$

where the drag coefficients $\Delta_{n}$ and $\Lambda_{n}$ are associated with harmonic translational or rotational motion of a sphere in unsteady viscous flows (Kim \& Karrila 2005, see also $\S 3.4)$

$$
\Delta_{n}=6 \pi\left(1+n^{1 / 2} \tilde{\lambda}+\frac{n \tilde{\lambda}^{2}}{9}\right) \text { and } \Lambda_{n}=8 \pi \frac{1+n^{1 / 2} \tilde{\lambda}+\mathrm{i} n \tilde{\lambda}^{2} / 3}{1+n^{1 / 2} \tilde{\lambda}}
$$

and $\tilde{\lambda}=\mathrm{e}^{\mathrm{i} \pi / 4} \lambda$.

The leading order dynamics $\left(\hat{v}_{0, y}, \hat{\theta}_{0}\right)$ is then obtained from the linear system:

$$
\begin{gathered}
{\left[\mathrm{i}+\left(\frac{3}{4 \pi} \frac{\beta}{\lambda^{2}}\right) \Delta_{1}\right] \hat{v}_{0, y}-\alpha \hat{\theta}_{0}=\mathrm{i}(\beta-1),} \\
\left(\frac{15}{8 \pi} \frac{\alpha \beta}{\lambda^{2} I}\right) \Delta_{1} \hat{v}_{0, y}+\left[1-\mathrm{i}\left(\frac{15}{8 \pi} \frac{\beta}{\lambda^{2} I}\right) \Lambda_{1}\right] \hat{\theta}_{0}=\mathrm{i}\left(\frac{5}{2} \frac{\alpha \beta}{I}\right) .
\end{gathered}
$$

It should be noted that the above dynamics is independent from that along the $x$ direction, which can be computed in a second step. The complex amplitude of the angular velocity $\hat{\omega}_{0}$ is then obtained using $\hat{\omega}_{0}=\mathrm{i} \varepsilon \hat{\theta}_{0}$.

From the small and large $\lambda$ approximations of $\Delta_{1}$ and $\Lambda_{1}$, Eqs. (2.14)-(2.15) can be 


\begin{tabular}{ccc} 
parameters & expression & physical meaning \\
\hline$\beta$ & $\rho / \rho_{s}$ & fluid-to-solid density ratio \\
$\varepsilon$ & $\xi / a$ & dimensionless displacement amplitude of the acoustic field \\
$\alpha$ & $\delta / a$ & dimensionless imbalance parameter \\
$I$ & $I_{G} / I_{0}$ & dimensionless moment of inertia \\
$\lambda$ & $\left(\varpi a^{2} / \nu\right)^{1 / 2}$ & inverse of the dimensionless viscous length
\end{tabular}

TABLE 1. List of the five independent parameters of the problem. Note that the Reynolds number $\operatorname{Re}=\varepsilon \lambda^{2}$, which is supposed to be small compared to unity, is not an independent parameter.

used to obtain the following useful asymptotic forms of $\hat{v}_{0, y}$ and $\hat{\theta}_{0}$ :

$$
\begin{gathered}
\hat{v}_{0, y} \sim \frac{2 \mathrm{i} \lambda^{2}(\beta-1)}{9 \beta} \text { and } \hat{\theta}_{0} \sim-\frac{\alpha \lambda^{2}}{6 \beta} \text { for } \lambda \rightarrow 0, \\
\hat{v}_{0, y} \sim \frac{4 I(\beta-1)+10 \alpha^{2} \beta}{4 I+\beta\left(2 I+5 \alpha^{2}\right)} \text { and } \hat{\theta}_{0} \sim \frac{15 \mathrm{i} \alpha \beta}{4 I+\beta\left(2 I+5 \alpha^{2}\right)} \text { for } \lambda \rightarrow \infty .
\end{gathered}
$$

\section{Acoustic propulsion of the sphere}

Knowing the leading-order viscous response of the sphere to the incident acoustic field, we now proceed to explore the possibility to achieve propulsion by means of streaming effects, by accounting for the first inertial correction to the flow field following the approach of Lippera et al. (2019).

\subsection{Governing equations}

By moving through the fluid, the sphere generates a flow field $\boldsymbol{u}(\boldsymbol{r}, t)$ around itself governed by the Navier-Stokes and continuity equations, which can be written in nondimensional form in the frame of reference moving with the fluid far from the sphere as

$$
\lambda^{2} \frac{\partial \boldsymbol{u}}{\partial t}+\operatorname{Re} \boldsymbol{\nabla} \boldsymbol{u} \cdot \boldsymbol{u}=\boldsymbol{\nabla} \cdot \boldsymbol{\sigma}, \boldsymbol{\nabla} \cdot \boldsymbol{u}=0,
$$

where $\boldsymbol{\sigma}$ is the non-dimensional hydrodynamic stress in the fluid due to the relative motion between the sphere and the surrounding fluid (and therefore includes a corrected pressure to account for the inertial corrections associated with the moving frame). It is recalled that, as in the previous section, all quantities are non-dimensional and $a, \varpi^{-1}$, and $\xi \varpi$ are used as reference length, time and velocity scales respectively. In Eq. (3.1), the Reynolds number is $\operatorname{Re}=\varepsilon \lambda^{2}$ with $\varepsilon=\xi / a \ll 1$. In the following we thus focus on the limit of $\operatorname{Re} \ll 1$, which yields the restriction $\lambda^{2} \ll \varepsilon^{-1}$ for the following analysis. Note that $\mathrm{Re}$ is therefore not a new independent dimensionless group so that the problem is only governed by the five parameters $\varepsilon, \lambda, \alpha, \beta$ and $I$ defined in the previous section, and listed in table 1.

The flow field vanishes at infinity and satisfies the no-slip boundary condition on the moving sphere $(|\boldsymbol{r}|=1$ in a set of axes attached to the centroid of the sphere), therefore

$$
\boldsymbol{u}=\boldsymbol{v}+\boldsymbol{\omega} \times \boldsymbol{r} \text { for }|\boldsymbol{r}|=1, \quad \boldsymbol{u} \rightarrow 0 \text { for }|\boldsymbol{r}| \rightarrow \infty .
$$

\subsection{Expansions in power of Re and order of the propulsion speed}

The Reynolds number Re is a small parameter of the problem, and we now expand the velocity field $\boldsymbol{u}$, the hydrodynamic stress $\boldsymbol{\sigma}$, and the velocity of the sphere $\boldsymbol{v}$ in powers 
of the Reynolds number

$$
\boldsymbol{u}=\boldsymbol{u}^{(0)}+\operatorname{Re} \boldsymbol{u}^{(1)}+\cdots, \quad \boldsymbol{\sigma}=\boldsymbol{\sigma}^{(0)}+\operatorname{Re} \boldsymbol{\sigma}^{(1)}+\cdots, \quad \boldsymbol{v}=\boldsymbol{v}^{(0)}+\operatorname{Re} \boldsymbol{v}^{(1)}+\cdots .
$$

We are interested in the emergence of a net propulsion of the sphere and therefore will focus on the existence of a steady component to the sphere's velocity. Due to the linearity of the unsteady Stokes equation, such steady motions have to be generated at order $O(\operatorname{Re})$ at least, which can be written $\overline{\boldsymbol{v}}=\operatorname{Re}\left\langle\boldsymbol{v}^{(1)}\right\rangle$, where $\langle\cdots\rangle$ refers to the time average operator over a period of oscillation. In other words, the possibly non-zero $O(\operatorname{Re})$ steady component of the speed $\overline{\boldsymbol{v}}$ must be induced by the steady streaming flow resulting from the self-coupling of the $O(1)$ (i.e. $\mathrm{Re}=0$ ) viscous flow through the nonlinear term of the Navier-Stokes equation.

To obtain such a forcing, one could explicitly derive the steady streaming flow and integrate the corresponding hydrodynamic stress over the surface of the sphere. In order to circumvent such a cumbersome derivation, we use in the following a specific form of Lorentz reciprocal theorem suitable for the case where inertial corrections are considered (Ho \& Leal 1974; Nadal \& Lauga 2014; Lippera et al. 2019).

\subsection{Lorentz reciprocal theorem for inertial corrections}

To this end, we define the auxiliary flow and stress fields $\left(\boldsymbol{u}^{\star}, \boldsymbol{\sigma}^{\star}\right)$, as the unique solution of the following steady Stokes problem

$$
\boldsymbol{\nabla} \cdot \boldsymbol{\sigma}^{\star}=\mathbf{0} \text { and } \boldsymbol{\nabla} \cdot \boldsymbol{u}^{\star}=0
$$

with boundary conditions

$$
\boldsymbol{u}^{\star}=\boldsymbol{v}^{\star}+\boldsymbol{\omega}^{\star} \times \boldsymbol{r} \text { at }|\boldsymbol{r}|=1, \quad \boldsymbol{u}^{\star} \rightarrow 0 \text { for }|\boldsymbol{r}| \rightarrow \infty .
$$

Using Eqs. (3.1) and (3.4) and denoting by $\mathcal{V}$ the volume of fluid outside the sphere, one can write an instantaneous version of the Lorentz reciprocal theorem (for further details, see again Lippera et al. 2019) in the following form:

$$
\lambda^{2} \int_{\mathcal{V}} \boldsymbol{u}^{\star} \cdot \frac{\partial \boldsymbol{u}}{\partial t} d \mathcal{V}+\operatorname{Re} \int_{\mathcal{V}}\left[\boldsymbol{u}^{\star} \cdot \nabla \boldsymbol{u} \cdot \boldsymbol{u}\right] d \mathcal{V}=\boldsymbol{f}^{\star} \cdot \boldsymbol{v}+\boldsymbol{l}^{\star} \cdot \boldsymbol{\omega}-\boldsymbol{v}^{\star} \cdot \boldsymbol{f}-\boldsymbol{\omega}^{\star} \cdot \boldsymbol{l} .
$$

where $\boldsymbol{f}$ and $\boldsymbol{l}$ (resp. $\boldsymbol{f}^{\star}$ and $\boldsymbol{l}^{\star}$ ) are the hydrodynamic force and torque in $O$ for the real (resp. auxiliary) problem. Because the particle is spherical, we immediately have $\boldsymbol{f}^{\star}=-6 \pi \boldsymbol{v}^{\star}$ and $\boldsymbol{l}^{\star}=-8 \pi \boldsymbol{\omega}^{\star}$ and Eq. (3.6) becomes

$$
-(6 \pi \boldsymbol{v}+\boldsymbol{f}) \cdot \boldsymbol{v}^{\star}-(8 \pi \boldsymbol{\omega}+\boldsymbol{l}) \cdot \boldsymbol{\omega}^{\star}=\lambda^{2} \frac{d}{d t}\left[\int_{\mathcal{V}} \boldsymbol{u}^{\star} \cdot \boldsymbol{u} d \mathcal{V}\right]+\operatorname{Re} \int_{\mathcal{V}}\left[\boldsymbol{u}^{\star} \cdot \nabla \boldsymbol{u} \cdot \boldsymbol{u}\right] d \mathcal{V},
$$

since $\boldsymbol{u}^{\star}$ is time-independent and $\mathcal{V}$ is fixed in time. It should be noted that up until now, no assumption on the magnitude of Re was used and the previous equation is therefore valid for any value of the Reynolds number.

Now, introducing Eqs. (3.3) and the additional Re-expansions

$$
\boldsymbol{f}=\boldsymbol{f}^{(0)}+\operatorname{Re} \boldsymbol{f}^{(1)}+\cdots, \quad \boldsymbol{l}=\boldsymbol{l}^{(0)}+\operatorname{Re} \boldsymbol{l}^{(1)}+\cdots .
$$

for the force and torque into Eq. (3.7), and and identifying the $O(1)$ terms, leads to

$$
-\left(6 \pi \boldsymbol{v}^{(0)}+\boldsymbol{f}^{(0)}\right) \cdot \boldsymbol{v}^{\star}-\left(8 \pi \boldsymbol{\omega}^{(0)}+\boldsymbol{l}^{(0)}\right) \cdot \boldsymbol{\omega}^{\star}=\lambda^{2} \int_{\mathcal{V}} \boldsymbol{u}^{\star} \cdot \frac{\partial \boldsymbol{u}^{(0)}}{\partial t} d \mathcal{V} .
$$

Note that the right-hand side of Eq. (3.9) can be integrated provided assumptions on 
the harmonic nature of the $O(1)$ solution are formulated, in order to obtain the drag force and torque in unsteady Stokes flow $(\operatorname{Re}=0$, see $\S 3.4)$.

Considering now the $O(\operatorname{Re})$ terms in Eq. (3.7), the problem obtained at that order is structurally similar to that at $O(1)$ but for the emergence of an extra forcing that arises from and accounts for the effect of the streaming flow. Should a net self-propulsion occur (i.e. on average over a whole period of forcing), it would therefore be due to the streaming forcing, as anticipated. Taking the average in time of the resulting equation, one obtains

$$
-\boldsymbol{v}^{\star} \cdot\left\langle 6 \pi \boldsymbol{v}^{(1)}+\boldsymbol{f}^{(1)}\right\rangle-\boldsymbol{\omega}^{\star} \cdot\left\langle 8 \pi \boldsymbol{\omega}^{(1)}+\boldsymbol{l}^{(1)}\right\rangle=\left\langle\int_{\mathcal{V}}\left[\boldsymbol{u}^{\star} \cdot \nabla \boldsymbol{u}^{(0)} \cdot \boldsymbol{u}^{(0)}\right] d \mathcal{V}\right\rangle=\mathcal{H} .
$$

In order to derive the steady component of the propulsion speed $\overline{\boldsymbol{v}}=\operatorname{Re}\left\langle\boldsymbol{v}^{(1)}\right\rangle$, our goal in the following lies in the computation of the right-hand-side, $\mathcal{H}$, of the previous equality.

\subsection{Viscous drags and steady propulsion speed}

Knowing the form of the viscous dynamical response of the sphere $\left(\hat{\boldsymbol{v}}_{0}, \hat{\omega}_{0}\right)$ from $\S 2$, we are now able to derive an explicit expression of the propulsion speed $\overline{\boldsymbol{v}}$. We first write $\boldsymbol{v}^{(0)}=\hat{\boldsymbol{v}}_{0} \mathrm{e}^{\mathrm{i} t}, \boldsymbol{\omega}^{(0)}=\hat{\boldsymbol{\omega}}_{0} \mathrm{e}^{\mathrm{i} t}, \boldsymbol{u}^{(0)}=\hat{\boldsymbol{u}}_{0} \mathrm{e}^{\mathrm{i} t}, \boldsymbol{f}^{(0)}=\hat{\boldsymbol{f}}_{0} \mathrm{e}^{\mathrm{i} t}$ and $\boldsymbol{l}^{(0)}=\hat{\boldsymbol{l}}_{0} \mathrm{e}^{\mathrm{i} t}$.

In this context, the $O(1)$ and $O(\mathrm{Re})$ components of Eqs. (3.9) and (3.10) become

$$
\begin{gathered}
-6 \pi\left(\hat{\boldsymbol{v}}_{0}+\hat{\boldsymbol{f}}_{0}\right) \cdot \boldsymbol{v}^{\star}-\left(8 \pi \hat{\boldsymbol{\omega}}_{0}+\hat{\boldsymbol{l}}_{0}\right) \cdot \boldsymbol{\omega}^{\star}=\lambda^{2} \int_{\mathcal{V}} \boldsymbol{u}^{\star} \cdot \frac{\partial \hat{\boldsymbol{u}}_{0}}{\partial t} d \mathcal{V} \\
-\boldsymbol{v}^{\star} \cdot\left\langle 6 \pi \boldsymbol{v}^{(1)}+\boldsymbol{f}^{(1)}\right\rangle-\boldsymbol{\omega}^{\star} \cdot\left\langle 8 \pi \boldsymbol{\omega}^{(1)}+\boldsymbol{l}^{(1)}\right\rangle=\frac{1}{2} \Re\left\{\int_{\mathcal{V}}\left[\boldsymbol{u}^{\star} \cdot \boldsymbol{\nabla} \hat{\boldsymbol{u}}_{0}^{\dagger} \cdot \hat{\boldsymbol{u}}_{0}\right] d \mathcal{V}\right\}=\mathcal{H} .
\end{gathered}
$$

where $\Re(z)$ and $z^{\dagger}$ stand for the real part and complex conjugate of $z$.

In the case of an harmonic motion, $\hat{\boldsymbol{u}}_{0}$ is given by

$$
\hat{\boldsymbol{u}}_{0}=[A(r) \mathbf{I}+B(r) \mathbf{n n}] \cdot \hat{\boldsymbol{v}}_{0}+C(r) \hat{\boldsymbol{\omega}}_{0} \times \mathbf{n},
$$

where the exact forms for given $\lambda$ of $A(r), B(r)$ and $C(r)$ are reminded in Appendix A (see also chapter 6 in Kim \& Karrila 2005). The velocity field induced by a rectilinear steady motion of a sphere in a viscous fluid has a form similar to Eq. (3.13)

$$
\boldsymbol{u}^{\star}=\left[A^{\star}(r) \mathbf{I}+B^{\star}(r) \mathbf{n n}\right] \cdot \boldsymbol{v}^{\star}+C^{\star}(r) \boldsymbol{\omega}^{\star} \times \mathbf{n},
$$

where the exact forms of $A^{\star}(r), B^{\star}(r)$ and $C^{\star}(r)$ are also given in appendix A, and are in fact respectively the asymptotic limits of $A, B$ and $C$ for $\lambda \rightarrow 0$ (steady motion).

\subsubsection{Order $O(1)$ - Viscous response}

Successively introducing the auxiliary fields $\left(\boldsymbol{v}^{\star}, \boldsymbol{\omega}^{\star}\right)=\left(\mathbf{e}_{y}, \mathbf{0}\right)$ and $\left(\boldsymbol{v}^{\star}, \boldsymbol{\omega}^{\star}\right)=\left(\mathbf{0}, \mathbf{e}_{z}\right)$ in Eq. (3.11) provides

$$
\hat{\boldsymbol{f}}_{0}=-\left[6 \pi+\tilde{\lambda}^{2} F(\lambda)\right] \hat{\boldsymbol{v}}_{0}, \quad \hat{\boldsymbol{l}}_{0}=-\left[8 \pi+\tilde{\lambda}^{2} G(\lambda)\right] \hat{\boldsymbol{\omega}}_{0}
$$

with

$$
F(\lambda)=4 \pi \int_{1}^{\infty} r^{2}\left[\frac{2 A A^{\star}+(A+B)\left(A^{\star}+B^{\star}\right)}{3}\right] d r, \quad G(\lambda)=\frac{8 \pi}{3} \int_{1}^{\infty} r^{2} C C^{\star} d r
$$

and $\tilde{\lambda}^{2}=\mathrm{i} \lambda^{2}$. One can note that computing the integral on the right-hand sides of Eqs. (3.16) indeed provides the classical expressions derived for the unsteady translational 

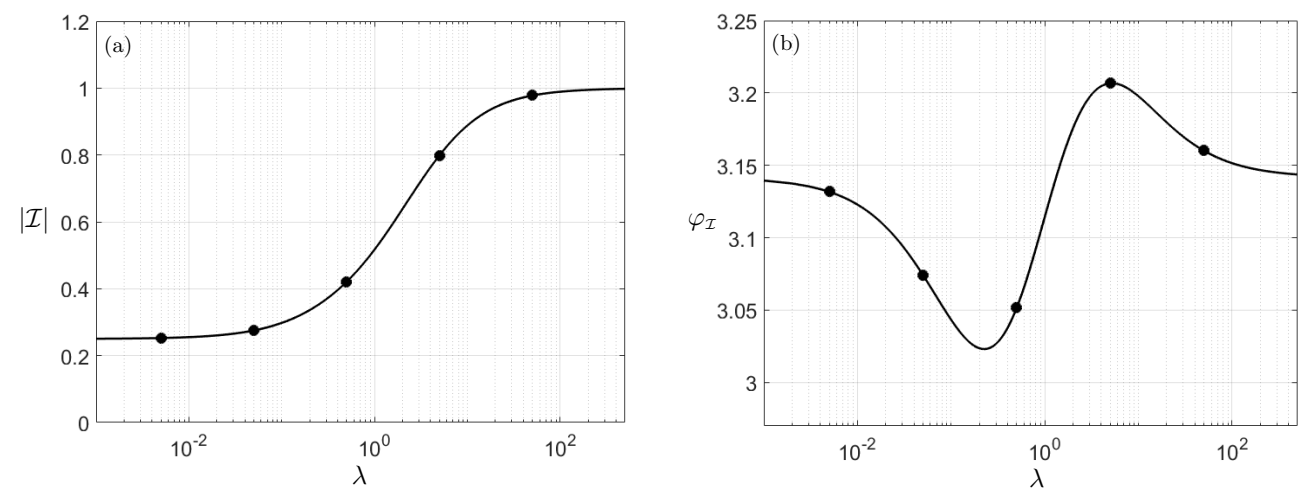

Figure 2. Magnitude (a) and phase (b) of $\mathcal{I}$. The bullets correspond to the direct numerical integration of Eq. (3.20).

and rotational drag (Stokes 1850; Mazur \& Bedeaux 1974; Kim \& Karrila 2005):

$$
\hat{\boldsymbol{f}}_{0}=-6 \pi\left(1+\tilde{\lambda}+\frac{\tilde{\lambda}^{2}}{9}\right) \hat{\boldsymbol{v}}_{0}, \quad \hat{\boldsymbol{l}}_{0}=-8 \pi \frac{1+\tilde{\lambda}+\tilde{\lambda}^{2} / 3}{1+\tilde{\lambda}} \hat{\boldsymbol{\omega}}_{0} .
$$

\subsubsection{Order $O(R e)$ - Propulsion speed}

Let us turn to the leading-order mean propulsion speed $\overline{\boldsymbol{v}}$. Using Eq. (3.13),

$$
\begin{aligned}
\boldsymbol{\nabla} \hat{\boldsymbol{u}}_{0}=A^{\prime} \hat{\boldsymbol{v}}_{0} \mathbf{n}+B^{\prime}\left(\mathbf{n} \cdot \hat{\boldsymbol{v}}_{0}\right) \mathbf{n n}+ & \frac{B}{r}\left\{\left(\mathbf{n} \cdot \hat{\boldsymbol{v}}_{0}\right)(\mathbf{I}-\mathbf{n n})+\mathbf{n} \otimes\left[(\mathbf{I}-\mathbf{n n}) \cdot \hat{\boldsymbol{v}}_{0}\right]\right\} \\
+ & +C^{\prime}\left(\hat{\boldsymbol{\omega}}_{0} \times \mathbf{n}\right) \otimes \mathbf{n}-\frac{C}{r}\left[\boldsymbol{\epsilon} \cdot \hat{\boldsymbol{\omega}}_{0}+\left(\hat{\boldsymbol{\omega}}_{0} \times \mathbf{n}\right) \otimes \mathbf{n}\right],
\end{aligned}
$$

where $C^{\prime}=d C / d r$ and $(\boldsymbol{\epsilon})_{i j k}=\epsilon_{i j k}$, so that $\left(\boldsymbol{\epsilon} \cdot \boldsymbol{\omega}_{0}\right) \cdot \boldsymbol{a}=\boldsymbol{a} \times \boldsymbol{\omega}_{0}$ for any vector $\boldsymbol{a}$. Introducing Eqs. (3.13), (3.14) and (3.18) in Eq. (3.12) and performing the explicit integration of its right-hand side leads to

$$
\mathcal{H}=\frac{2 \pi}{3} \Re\left\{\left[\boldsymbol{v}^{\star} \cdot\left(\hat{\boldsymbol{\omega}}_{0} \times \hat{\boldsymbol{v}}_{0}^{\dagger}\right)\right] \mathcal{I}(\tilde{\lambda})\right\},
$$

where the quantity

$$
\mathcal{I}(\tilde{\lambda})=\int_{1}^{\infty}\left[A^{\star}\left(A^{\dagger} C^{\prime}+B^{\dagger} C^{\prime}+\frac{2 A^{\dagger} C}{r}\right)+\frac{B^{\star}\left(A^{\dagger}-B^{\dagger}\right) C}{r}\right] r^{2} d r
$$

is given in its fully integrated form in appendix B and its variations are indicated on Figure 2. In particular, for small and large $\lambda$, the asymptotic behaviour of $\mathcal{I}$ is obtained as

$$
\mathcal{I}(\lambda \rightarrow 0)=-\frac{1}{4}, \quad \mathcal{I}(\lambda \rightarrow \infty)=-1 .
$$

Note that Eq. (3.19) confirms that the $x$-component of $\hat{\boldsymbol{v}}_{0}$ will have no contribution to the steady motion, as anticipated in $\S 2$ and expected for symmetry reasons.

Now, choosing $\boldsymbol{v}^{\star}=\mathbf{e}_{x}$ and $\boldsymbol{\omega}^{\star}=\mathbf{0}$ in Eqs. (3.12) and (3.19), and remembering that (i) using Eq. (2.11), only the $y$-component of $\hat{\boldsymbol{v}}_{0}$ has a non zero contribution to the mean propulsion speed, (ii) $\hat{\boldsymbol{\omega}}_{0}$ is along the $z$-axis, and (iii) $\langle\boldsymbol{f}\rangle=0$ due to the periodicity of 
10

$\left\langle v^{(1)}\right\rangle / \varepsilon$

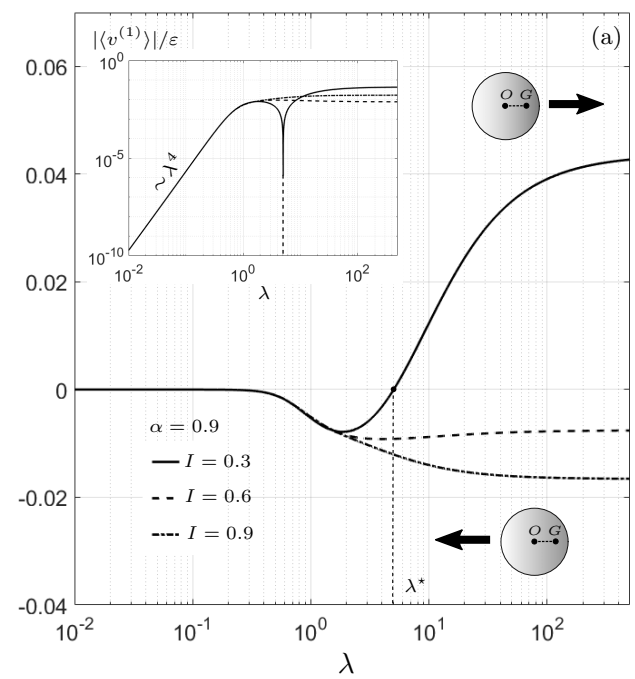

$\left\langle v^{(1)}\right\rangle / \varepsilon$

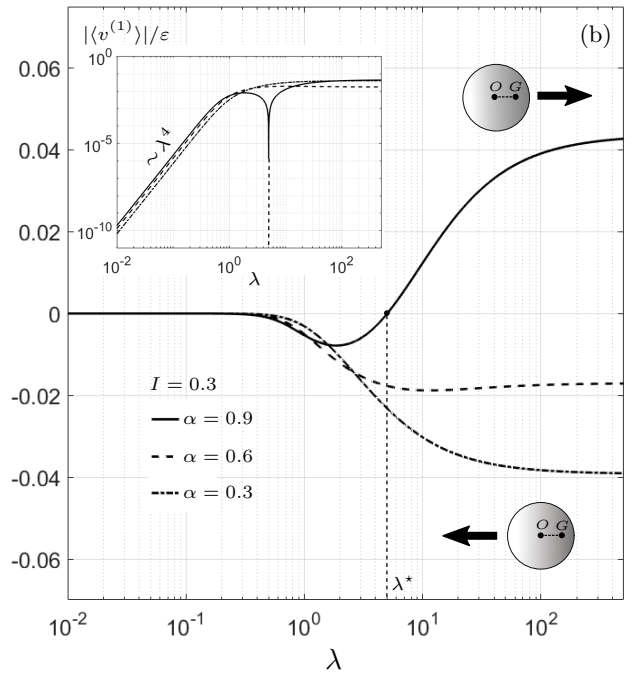

Figure 3. Ratio $\left\langle v^{(1)}\right\rangle / \varepsilon$ as a function of $\lambda$ for $\beta=0.2$ and several combinations of $(I, \alpha)$. (a) $\alpha=0.9, I=0.3,0.6,0.9$; (b) $I=0.3, \alpha=0.3,0.6,0.9$. The spheres sketched in (a) and (b) illustrate the direction of propulsion when the center of mass is on the right of the geometric center (top: $\left\langle v^{(1)}\right\rangle / \varepsilon>0$; bottom: $\left\langle v^{(1)}\right\rangle / \varepsilon<0$ ).

the particle's velocity, one obtains

$$
\left\langle\boldsymbol{v}^{(1)}\right\rangle=\left\langle v^{(1)}\right\rangle \mathbf{e}_{x}=\frac{1}{9} \Re\left[\hat{\omega}_{0} \hat{v}_{0, y}^{\dagger} \mathcal{I}(\tilde{\lambda})\right] \mathbf{e}_{x},
$$

or equivalently, as a function of the tilt angle amplitude,

$$
\left\langle\boldsymbol{v}^{(1)}\right\rangle=-\frac{1}{9} \varepsilon \Im\left[\hat{\theta}_{0} \hat{v}_{0, y}^{\dagger} \mathcal{I}(\tilde{\lambda})\right] \mathbf{e}_{x},
$$

where $\Im(z)$ refers to the imaginary part of $z$.

Note that the ratio $\left\langle v^{(1)}\right\rangle / \varepsilon$, which is a function of the four dimensionless parameters $\alpha, \beta, I$ and $\lambda$, does not depend on $\varepsilon$. As a result, the leading order dimensionless mean velocity of the particle $\overline{\boldsymbol{v}}$ is the product of $\varepsilon$ Re and of a dimensionless function of the four other parameters. The quantity $\left\langle v^{(1)}\right\rangle / \varepsilon$ is plotted in Fig. 3 for $\beta=0.2$ and different combinations $(I, \alpha)$.

\subsection{Asymptotic behaviour and reversal of the propulsion speed}

As shown on Fig. 3, for large $\alpha$ or small $I$, a reversal of the direction of propulsion (illustrated by the diagrams inserted in each sub-figure) can be observed at a finite value $\lambda^{\star}$ of the reduced frequency $\lambda$. This reversal in swimming direction is not the result of the difference in behaviour of the streaming flows at low and high frequencies, and is instead entirely due to a change by a factor of $\pi$ in the relative phase between translation and rotation in the viscous (i.e. $\mathrm{Re}=0$ ) response of the forced sphere.

The variations of $\lambda^{\star}(\beta, I, \alpha)$ are plotted in Fig. 4, for three different values of the density ratio $\beta$. In each case, the $(I, \alpha)$-plane is divided into two regions: a first one where a reversal of the direction of propulsion can be observed at finite $\lambda$, and another one, where the direction of propulsion does not depend on $\lambda$ (in the latter case, the sphere always propels with the light end ahead). The limit between the two regions (i.e. a criterion for existence of the reversal in swimming direction between small and large 

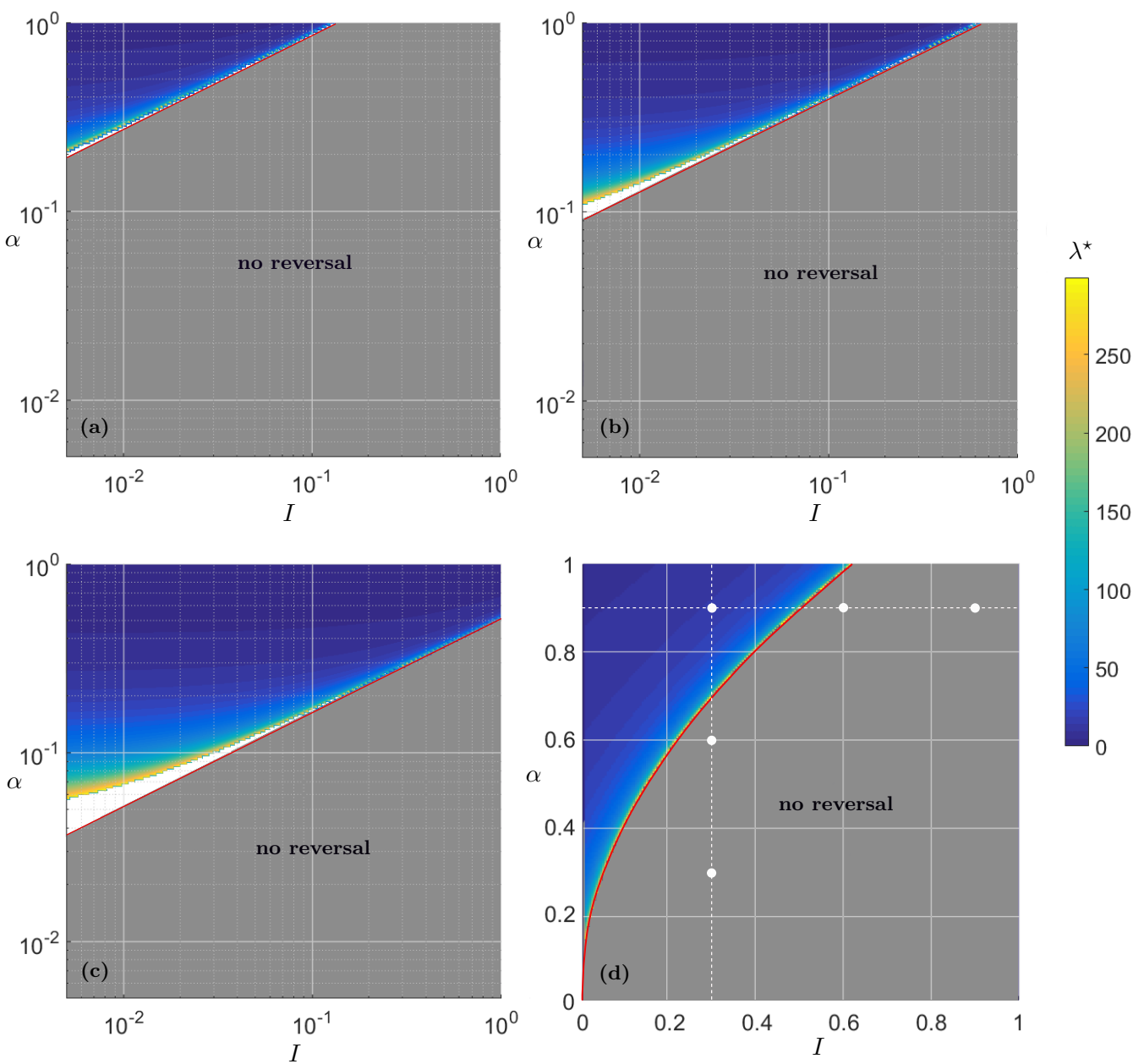

Figure 4 . Value $\lambda^{\star}$ corresponding to a reversal of the propulsion direction plotted in the plane $(I, \alpha)$ for three values of $\beta$. (a) $\beta=0.05$; (b,d) $\beta=0.2$ - figure (d) is the same as (b) but plotted in a linear scale ; (c) $\beta=0.6$. For a fixed value of $\beta$, the frontier between the reversal and the non-reversal regions is given by the equality case of Eq. (3.26) (solid red line). The cases considered in Fig. 3 are specified on (d) using white bullets. The color bar (top right) holds for all the figures. The white zones in Figs (a), (b) and (c), where $\lambda^{\star}$ is not computed, comes from the practical limitation in the numerical extraction of $\lambda^{\star}$ which tends to infinity in the vicinity of the transition (see text fo further explanation).

$\lambda$ ) can be obtained by deriving the asymptotic behaviour of $\left\langle v^{(1)}\right\rangle$ at small and large $\lambda$. Substituting the result of Eqs. (2.16), (2.17) and (3.21) into Eq. (3.23), one obtains

$$
\begin{gathered}
\left\langle v^{(1)}\right\rangle \sim \frac{\varepsilon \alpha \lambda^{4}(\beta-1)}{972 \beta^{2}} \text { for } \lambda \rightarrow 0, \\
\left\langle v^{(1)}\right\rangle \sim \frac{10 \varepsilon \alpha \beta\left[2 I(\beta-1)+5 \alpha^{2} \beta\right]}{3\left[4 I+\beta\left(2 I+5 \alpha^{2}\right)\right]^{2}} \text { for } \lambda \rightarrow \infty .
\end{gathered}
$$

A change in swimming direction between the $\lambda \ll 1$ and $\lambda \gg 1$ limits therefore requires 
$\beta-1$ and $2 I(\beta-1)+5 \alpha^{2} \beta$ to have opposite signs, or equivalently

$$
0 \leqslant \frac{2(1-\beta)}{5 \beta} \leqslant \frac{\alpha^{2}}{I}
$$

This is consistent with the results shown in Fig. 4 where the red line corresponds to the equality case above. The presence of a white zone in Figs. 4a-c, where $\lambda^{\star}$ is not computed, comes from the practical limitation in the numerical extraction of $\lambda^{\star}$ which tends to infinty in the vicinity of the transition region (red line). This region would be reduced if the upper bound of the research interval in $\lambda$ was enlarged. This has been verified for the value $\beta=0.05$, for which the white zone barely exists. Note that the reversal is only possible if $\beta \leqslant 1$ (i.e. the particle must be heavier than the fluid on average) and if a sufficiently large inhomogeneity exists (as measured by $\alpha$ ).

\section{Physical discussion and orders of magnitude}

The dimensional form of Eq. (3.23) is

$$
\bar{V}=\xi \varpi \bar{v}=-\frac{\xi^{3} \varpi^{2}}{9 \nu} \Im\left[\hat{\theta}_{0} \hat{v}_{0}^{\dagger} \mathcal{I}(\tilde{\lambda})\right],
$$

where it should be noted that the radius of the particle only appears through $\lambda$ (and not in the pre-factor).

Based on a mean value of the quality factor of the acoustic cavity $Q \sim 300$ (see Bruss 2012) and a typical displacement of the piezoelectric wall $\ell \sim 0.1 \mathrm{~nm}$, a maximum value for the displacement amplitude at the pressure node can be estimated as $\xi=$ $2 Q \ell / \pi \sim 19 \mathrm{~nm}$. For a typical particle radius $a=0.5 \mu \mathrm{m}$ and forcing frequency of $4 \mathrm{MHz}$, corresponding respectively to $\varepsilon=0.038$ and $\lambda \simeq 2.5$, a value of $\left|\left\langle v^{(1)}\right\rangle\right| / \varepsilon \sim 0.01$ is a reasonable estimate of the particle's dimensionless velocity (see Fig. 3) and one obtains dimensionally

$$
\bar{V} \sim 44 \mu \mathrm{m} \mathrm{s}^{-1},
$$

which is consistent with the values reported by Ahmed et al. (2016). A quality factor of $10^{3}$ (upper bound measured in standard acoustic resonators, see again Bruss 2012) would have led to a propulsion velocity $\bar{V} \simeq 1.6 \mathrm{~mm} \mathrm{~s}^{-1}$, which is much larger than the values reported by Ahmed et al. (2016) or Wang et al. (2012) (the latter reports a maximum value of $200 \mu \mathrm{m} \mathrm{s}^{-1}$ ), but is not inconsistent with the velocities measured by Kaynak et al. (2017). A quality factor of $10^{2}$ (lower bound measured in standard acoustic resonators) would yield $\bar{V} \simeq 1.6 \mu \mathrm{m} \mathrm{s}^{-1}$.

In brief, even if the orders of magnitude of propulsion speed produced by the model are not irrelevant to the measurements, performing a quantitative comparison remains difficult because (i) the spherical geometry of our model noticeably departs from the experimental geometry depicted in Wang et al. (2012) and Ahmed et al. (2016) and (ii) the quality factor of the experimental acoustic cavities used by Wang et al. (2012) and Ahmed et al. (2016) is not known, whereas it critically impacts the estimate of the velocity. The profile of the quantity $\left\langle v^{(1)}\right\rangle / \varepsilon$ with respect to the density ratio $\beta$, for $\lambda=2.5, I=0.9$ and different values of the parameter $\alpha$ is plotted in Fig. 5. As mentioned by Ahmed et al. (2016), homogeneous Rhodium rods $(\beta=0.081)$ were faster than heavier golden ones $(\beta=0.052)$, an observation which is again consistent with the values presented in the figure.

A final practical yet fundamental remark must be made regarding the zero mean value of the tilt angle. Indeed, we assumed here that the tilt angle varied periodically around 


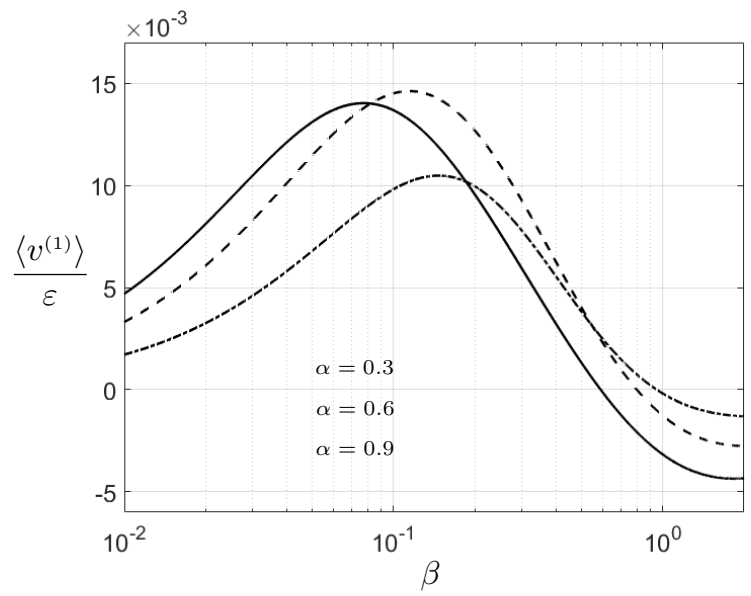

Figure 5. (a) Ratio $\left\langle v^{(1)}\right\rangle / \varepsilon$ as a functions of $\beta$ for $\lambda=2.5, I=0.9$ and different values of $\alpha$.

the value $\theta=0$ in the permanent regime (no angular drift). This assumption is not $a$ priori fully-justified since the radiation pressure on a sphere has no obvious orientation effect. In contrast, a near-sphere or an ellipsoid will orient itself in such a way that, on average, its major axis would lie in the zero-pressure plane of the wave. Therefore, the present calculation can be seen as the leading order calculation of the acoustic propulsion of a non-homogeneous near-sphere, since a slight alteration of the shape would not modify the propulsion speed obtained at leading order for a non-homogeneous sphere.

\section{Conclusion}

We present here a full derivation of the acoustic propulsion speed of a non-homogeneous rigid sphere. Unlike previous studies which generally rely on a numerically-integrated result, the final result obtained by means of the inertial version of the Lorentz reciprocal theorem is integrated analytically. The problem is ruled by five independent dimensionless parameters: the in-homogeneity ratio or imbalance distance $\alpha$, the fluid/solid density ratio $\beta$, the dimensionless moment of inertia $I$, the dimensionless forcing amplitude $\varepsilon$ and the reduced frequency $\lambda^{2}$. For a given density ratio $\beta \leqslant 1$, a limit value $\lambda^{\star}$ of the parameter $\lambda$ may exist such that propulsion takes place in different directions at low frequency $\left(\lambda<\lambda^{\star}\right)$ and high frequency $\left(\lambda>\lambda^{\star}\right)$. A necessary and sufficient condition for the existence of a reversal in the propulsion direction for varying $\lambda$ was obtained as $0 \leqslant(2 / 5)[(1-\beta) / \beta] \leqslant \alpha^{2} / I$.

The trends of the propulsion speed as a function of $\lambda$ as well as the possible existence of a change in propulsion direction for $\lambda=\lambda^{\star}$ are fully consistent with the results published by Collis et al. (2017). As expected, in a case where the reversal value $\lambda^{\star}$ does exist (see Fig. 3), propulsion occurs at low frequency ( small $\lambda$ ) in the direction of the lighter part of the sphere (center of mass behind the centroid) whereas at higher frequency (large $\lambda$ ), the inhomogeneous sphere propels with the center of mass ahead. The dependence of the propulsion speed amplitude upon the density ratio is non monotonous and at high mean densities (typically for $\beta<0.1$ ), light particles propel faster than heavier ones. Yet, one should be cautious in connecting this result to the observation reported by Ahmed et al. (2016) on density effects. Indeed, Ahmed et al. (2016) report that lighter 
rods propel faster than denser ones, but the rods also display a geometric asymmetry which could play a central role as well. In order to test more thoroughly the model, dedicated experiments performed using low aspect ratio solid particles with controlled density inhomogeneities would be enlightening.

\section{Acknowledgments}

This project has received funding from the European Research Council (ERC) under the European Union's Horizon 2020 research and innovation programme under Grant Agreement 714027 (SM). The authors also acknowledge insightful discussions with K. Lippera, M. Benzaquen and E. Lauga on the problem.

\section{Appendix A. Definition of the coefficients $A, A^{\star}, B, B^{\star}, C$ and $C^{\star}$}

The full expressions of the coefficients $A, B$ and $C$ of the unsteady harmonic Stokes flow in Eq. (3.13) are given by

$$
\begin{aligned}
& A(r)=\frac{3}{2 \tilde{\lambda}^{2} r^{3}}\left[\left(1+\tilde{\lambda} r+\tilde{\lambda}^{2} r^{2}\right) \mathrm{e}^{\tilde{\lambda}(1-r)}-1-\tilde{\lambda}-\frac{\tilde{\lambda}^{2}}{3}\right] \\
& B(r)=\frac{3}{2 \tilde{\lambda}^{2} r^{3}}\left[3+3 \tilde{\lambda}+\tilde{\lambda}^{2}-\left(3+3 \tilde{\lambda} r+\tilde{\lambda}^{2} r^{2}\right) \mathrm{e}^{\tilde{\lambda}(1-r)}\right] \\
& C(r)=\frac{\mathrm{e}^{\tilde{\lambda}(1-r)}(1+\tilde{\lambda} r)}{(1+\tilde{\lambda}) r^{2}}
\end{aligned}
$$

where $\tilde{\lambda}=\lambda \mathrm{e}^{\mathrm{i} \pi / 4}$, and the corresponding coefficients $A^{\star}, B^{\star}$ and $C^{\star}$ of the auxiliary steady Stokes flow in Eq. (3.14) are given by

$$
A^{\star}(r)=\frac{3}{4 r}+\frac{1}{4 r^{3}}, \quad B^{\star}(r)=\frac{3}{4 r}-\frac{3}{4 r^{3}}, \quad C^{\star}(r)=\frac{1}{r^{2}} .
$$

\section{Appendix B. Integration of the streaming term $\mathcal{H}$ in the harmonic case}

We note here $J(r)$ the integrand in the right-hand side of Eq. (3.20), namely

$$
J(r)=\left[A^{\star}\left(A^{\dagger} C^{\prime}+B^{\dagger} C^{\prime}+\frac{2 A^{\dagger} C}{r}\right)+\frac{B^{\star}\left(A^{\dagger}-B^{\dagger}\right) C}{r}\right] r^{2}
$$

which can be rewritten explicitly as

$$
\begin{aligned}
& J(r)=\frac{1}{4 \tilde{\lambda}^{\dagger 2}(1+\tilde{\lambda})}\left[\mathrm{e}^{\tilde{\lambda}(1-r)}\left(\tilde{\lambda}^{\dagger 2}+3 \tilde{\lambda}^{\dagger}+3\right)\left(-\frac{3 \tilde{\lambda}^{2}}{r^{3}}-\frac{15 \tilde{\lambda}}{r^{4}}+\frac{3 \tilde{\lambda}}{r^{6}}+\frac{3}{r^{7}}-\frac{\tilde{\lambda}^{2}+15}{r^{5}}\right)\right. \\
& +3 \mathrm{e}^{\left(\tilde{\lambda}+\tilde{\lambda}^{\dagger}\right)(1-r)}\left(\frac{3 \tilde{\lambda} \tilde{\lambda}^{\dagger}\left(\tilde{\lambda}+2 \tilde{\lambda}^{\dagger}\right)}{r^{2}}+\frac{3\left(\tilde{\lambda}^{2}+5 \tilde{\lambda} \tilde{\lambda}^{\dagger}+2 \tilde{\lambda}^{\dagger 2}\right)}{r^{3}}\right. \\
& +\frac{\tilde{\lambda}^{2} \tilde{\lambda}^{\dagger}-2 \tilde{\lambda} \tilde{\lambda}^{\dagger 2}+15 \tilde{\lambda}+15 \tilde{\lambda}^{\dagger}}{r^{4}} \\
& \left.\left.+\frac{\tilde{\lambda}^{2}-3 \tilde{\lambda} \tilde{\lambda}^{\dagger}-2 \tilde{\lambda}^{\dagger 2}+15}{r^{5}}-\frac{3\left(\tilde{\lambda}+\tilde{\lambda}^{\dagger}\right)}{r^{6}}-\frac{3}{r^{7}}\right)\right]
\end{aligned}
$$


So that $\mathcal{I}(\tilde{\lambda})=\int_{1}^{\infty} J(r) \mathrm{d} r$ is obtained analytically as

$$
\begin{aligned}
\mathcal{I}(\tilde{\lambda})=\frac{1}{4 \tilde{\lambda}^{\dagger 2}(1+\tilde{\lambda})}\{ & \left(\tilde{\lambda}^{\dagger 2}+3 \tilde{\lambda}^{\dagger}+3\right)\left[-3 \tilde{\lambda}^{2} I_{3}-15 \tilde{\lambda} I_{4}+3 \tilde{\lambda} I_{6}+3 I_{7}-\left(\tilde{\lambda}^{2}+15\right) I_{5}\right] \\
& +3\left[3 \tilde{\lambda} \tilde{\lambda}^{\dagger}\left(\tilde{\lambda}+2 \tilde{\lambda}^{\dagger}\right) \tilde{I}_{2}+3\left(\tilde{\lambda}^{2}+5 \tilde{\lambda} \tilde{\lambda}^{\dagger}+2 \tilde{\lambda}^{\dagger 2}\right) \tilde{I}_{3}\right. \\
& +\left(\tilde{\lambda}^{2} \tilde{\lambda}^{\dagger}-2 \tilde{\lambda} \tilde{\lambda}^{\dagger 2}+15 \tilde{\lambda}+15 \tilde{\lambda}^{\dagger}\right) \tilde{I}_{4} \\
& \left.\left.+\left(\tilde{\lambda}^{2}-3 \tilde{\lambda} \tilde{\lambda}^{\dagger}-2 \tilde{\lambda}^{\dagger 2}+15\right) \tilde{I}_{5}-3\left(\tilde{\lambda}+\tilde{\lambda}^{\dagger}\right) \tilde{I}_{6}-3 \tilde{I}_{7}\right]\right\}
\end{aligned}
$$

with

$$
\begin{aligned}
& I_{1}=\int_{1}^{\infty} \frac{\mathrm{e}^{\tilde{\lambda}(1-r)}}{r} d r=\mathrm{e}^{\tilde{\lambda}} E_{1}(\tilde{\lambda}) \\
& \tilde{I}_{1}=\int_{1}^{\infty} \frac{\mathrm{e}^{\left(\tilde{\lambda}+\tilde{\lambda}^{\dagger}\right)(1-r)}}{r} d r=\mathrm{e}^{\left(\tilde{\lambda}+\tilde{\lambda}^{\dagger}\right)} E_{1}\left(\tilde{\lambda}+\tilde{\lambda}^{\dagger}\right) \\
& I_{n}=\int_{1}^{\infty} \frac{\mathrm{e}^{\tilde{\lambda}(1-r)}}{r^{n}} d r=\frac{1}{n-1}\left(1-\tilde{\lambda} I_{n-1}\right) \\
& \tilde{I}_{n}=\int_{1}^{\infty} \frac{\mathrm{e}^{\left(\tilde{\lambda}+\tilde{\lambda}^{\dagger}\right)(1-r)}}{r^{n}} d r=\frac{1}{n-1}\left[1-\left(\tilde{\lambda}+\tilde{\lambda}^{\dagger}\right) \tilde{I}_{n-1}\right]
\end{aligned}
$$

which are well defined since $\tilde{\lambda}$ has positive real part. In the above equation, $E_{1}(z)$ is the exponential integral (Abramowitz \& Stegun 1964). Using these results, one obtains $\mathcal{I}$ analytically for any $\lambda$ :

$$
\begin{aligned}
\mathcal{I}(\tilde{\lambda})= & \frac{1}{64 \tilde{\lambda}^{\dagger}(1+\tilde{\lambda})}\left[\left(\tilde{\lambda}^{\dagger}+3\right) \tilde{\lambda}^{5}-\tilde{\lambda}^{\dagger} \tilde{\lambda}^{4}+\left(2 \tilde{\lambda}^{\dagger}-18\right) \tilde{\lambda}^{3}\right. \\
& \left.-6 \tilde{\lambda}^{\dagger 2} \tilde{\lambda}^{2}+\left(-3 \tilde{\lambda}^{\dagger 3}+6 \tilde{\lambda}^{\dagger 2}-16 \tilde{\lambda}^{\dagger}\right) \tilde{\lambda}+3 \tilde{\lambda}^{\dagger 4}-3 \tilde{\lambda}^{\dagger 3}-48 \tilde{\lambda}^{\dagger 2}-16 \tilde{\lambda}^{\dagger}\right] \\
& -\frac{\mathrm{e}^{\tilde{\lambda}} E_{1}(\tilde{\lambda})}{64 \tilde{\lambda}^{\dagger 2}(1+\tilde{\lambda})}\left[\left(\tilde{\lambda}^{\dagger 2}+3 \tilde{\lambda}^{\dagger}+3\right)\left(\tilde{\lambda}^{6}-6 \tilde{\lambda}^{4}\right)\right] \\
& +\frac{3 \mathrm{e}^{\tilde{\lambda}+\tilde{\lambda}^{\dagger}} E_{1}\left(\tilde{\lambda}+\tilde{\lambda}^{\dagger}\right)}{64 \tilde{\lambda}^{\dagger 2}(1+\tilde{\lambda})}\left(\tilde{\lambda}+\tilde{\lambda}^{\dagger}\right)\left(\tilde{\lambda}-\tilde{\lambda}^{\dagger}\right)\left[\tilde{\lambda}^{4}-2 \tilde{\lambda}^{2} \tilde{\lambda}^{\dagger 2}-6 \tilde{\lambda}^{2}+\tilde{\lambda}^{\dagger 4}-18 \tilde{\lambda}^{\dagger 2}\right] .
\end{aligned}
$$

Real, imaginary part and phase of $\mathcal{I}$ are presented in Fig. 2. The asymptotic forms of $\mathcal{I}$ at small and large $\lambda$, Eq. (3.21), are obtained using the following asymptotic limits (Abramowitz \& Stegun 1964)

$$
E_{1}(z \rightarrow 0) \sim-\ln z-\gamma, \quad E_{1}(z \rightarrow \infty) \sim \frac{\mathrm{e}^{-z}}{z} .
$$

\section{REFERENCES}

Abramowitz, M. \& Stegun, I. A. 1964 Handbook of Mathematical Functions with Formulas, Graphs, and Mathematical Tables. New York: Dover.

Ahmed, S., Gentekos, D.T., Fink, C.A. \& Mallouk, T.E. 2014 Self-assembly of nanorod motors into geometrically regular multimers and their propulsion by ultrasound. $A C S$ Nano 8 (11), 11053-11060.

Ahmed, S., Wang, W., Bai, L., Gentekos, D.T., Hoyos, M. \& Mallouk, T.E. 2016 Density and shape effects in the acoustic propulsion of bimetallic nanorod motors. ACS Nano 10 (4), 4763-4769.

Anderson, J. L. 1989 Colloid Transport by Interfacial Forces. Annual Review of Fluid Mechanics 21, 61-99. 
Baraban, L., Streubel, R., Makarov, D., Han, L., D.Karnaushenko, Schmidt, O.G. \& Cuniberti, G. 2012 Fuel-Free Locomotion of Janus Motors: Magnetically Induced Thermophoresis. ACS Nano 7, 1360-1367.

Bruss, H. 2012 Acoustofluidics 2: Perturbation theory and ultrasound resonance modes. Lab Chip 12, 20-28.

Burdick, J., Laocharoenshuk, R., Wheat, P.M., Posner, J.D. \& Wang, J. 2008 Synthetic nanomotors in microchannel networks: Directional microchip motion and controlled manipulation of cargo. J. Am. Chem. Soc. 130, 8164-8165.

Campuzano, S., Kagan, D., Orozco, J. \& Wang, J. 2011 Motion-driven sensing and biosensing using electrochemically propelled nanomotors. Analyst 136, 4621-4630.

Collis, J., Jesse, F., Chakraborty, D. \& Sader, J.E. 2017 Autonomous propulsion of nanorods trapped in an acoustic field. Journal of Fluid Mechanics 825, 29-48.

Cordova-Figueroa, U.M. \& Brady, J.F. 2008 Osmotic propulsion: The osmotic motor. Phys. Rev. Lett. 100 (15), 158303.

Ebbens, S.J. \& Howse, J.R. 2010 In pursuit of propulsion at the nanoscale. Soft Matter 6, $726-738$.

Ebbens, S.J. \& Howse, J.R. 2011 Direct observation of the direction of motion for spherical catalytic swimmers. Langmuir 27, 12293-12296.

Golestanian, R., Liverpool, T.B. \& Ajdari, A. 2007 Designing phoretic micro- and nanoswimmers. New J. Phys. 9, 126.

Ho, B. P. \& LEAL, L. G. 1974 Inertial migration of rigid spheres in two-dimensional unidirectional flows. Journal of Fluid Mechanics 65 (2), 365400.

Ibele, M.E., Wang, Y., Kline, T.R., Mallouk, T.E. \& Sen, A. 2007 Hydrazine fuels for bimetallic catalytic microfluic pumping. J. Am. Chem. Soc. 129 (25), 7762-7763.

Jiang, H.R., Yoshinaga, N. \& SAno, M. 2010 Active motion of a janus particle by selfthermophoresis in a defocused laser beam. Phys. rev. Lett. 105, 268302.

Kaynak, M., Ozcelik, A., Nourhani, A., Lammert, P.E., Crespi, V. H. \& Huang, T. J. 2017 Acoustic actuation of bioinspired microswimmers. Lab Chip 17, 395-400.

Kim, S. \& Karrila, S.J. 2005 Microhydrodynamics. Dover publication, Inc.

Lauga, E. \& Powers, T. 2009 The hydrodynamics of swimming microorganisms. Rep. Prog. Phys. 72, 096601.

Lippera, K., Dauchot, O., Michelin, S., \& Benzaquen, M. 2019 No net motion for oscillating near-spheres at low Re numbers. J. Fluid. Mech 866, R1.

Mazur, P. \& Bedeaux, D. 1974 A generalization of Faxén's theorem to nonsteady motion of a sphere through an incompressible fluid in arbitrary flow. Physica D $\mathbf{7 6}, 235-246$.

NADAL, F. \& LAUGA, E. 2014 Asymmetric steady streaming as a mechanism for acoustic propulsion of rigid bodies. Phys. Fluids 26, 082001.

Nelson, B.J., Kaliakastos, I.K. \& Aввотt, J.J. 2010 Microrobots for minimally invasive medicine. Ann. Rev. Biomed. Eng. 12 (1), 041916.

Pavlick, R.A., Dey, K.K., Sirjoosingh, A., Benesi, A. \& Sen, A. 2013 A catalytically driven organometallic molecular motor. Nanoscale 5, 1301-1304.

Pavlick, R.A., Sengupta, S., McFadden, T., Zhang, H. \& Sen, A. 2011 A polymerization powered-motor. Angew. Chem. Int. Ed. 50, 9374-9377.

Paxton, W.F., Kistler, K.C., C.C., Olmeda, Sen, A., Angelo, S.K. St, Mallouk, Y., Thomas, E., Lammert, P.E. \& CRespi, V.H 2004 Catalytic nanomotors: Autonomous movement of stripped nanorods. J. Am. Chem. Soc. 126 (41), 13424-13431.

Purcell, E.M. 1977 Life at low reynolds number. Am. J. Phys. 45, 3-11.

Qian, B., Montiel, D., Bregulla, A., Cichos, F. \& Yang, H. 2013 Harnessing thermal fluctuations for purposeful activities: the manipulation of single microswimmers by adaptative photon nudging. Chem. Sci. 4, 1420-1429.

RILEy, N. 1966 On a sphere oscillating in a viscous fluid. Quart. Journ. Mech. and Applied Math. XIX (4), 461-472.

Sabrina, S., Tasinkevych, M., Ahmed, S., Brooks, A.M., Olvera de la Cruz, M., Mallouk, T.E. \& Bishop, K.J.M. 2018 Shape-directed microspinners powered by ultrasound. ACS Nano 12 (3), 2939-2947.

Smoluchowsky, M. 1921 Handbuch der Electrizitat und des Magnetismus. Graetz (ed.), Leipzig. 
Stokes, SiR G.G. 1850 On the effects of the internal friction of fluids on the motion of pendulums. Trans. of the Cambridge Phil. Soc. IX.

Sundararajan, S., Lammert, P.E., Zudans, A.W., Crespi, V.H. \& Sen, A. 2008 Catalytic motors for transport of colloidal cargo. Nano Lett. 8, 1271-1276.

Wang, W., Castro, L.A., Hoyos, M. \& Mallouk, T.E. 2012 Autonomous motion of metallic microrods propelled by ultrasound. ACS Nano 6 (7), 6122-6132.

Wang, W., Duan., W., Ahmed, S., Mallouk, T.E. \& Sen, A. 2013 Small power: Autonomous nano- and micromotors propelled by self-generated gradients. Nano Today 8, 531-554.

Wu, J., Kagan, S. Balasubramanian D., Manesh, K.M., Campuzano, S. \& Wang, J. 2010 Motion-based DNA Detection Using Catalytic Nanomotors. Nat. Commun. 1 (3), 36.

Zhang, W. \& Stone, H. A. 1998 Oscillatory motions of circular disks and nearly spherical particles in viscous flows. J. Fluid Mech. 367, 329-358. 